\title{
Probing ferroelectric behaviour in charge-transfer organic meta-nitroaniline
}

Dmitry Isakov', Semen Vasilev, Etelvina de Matos Gomes, Bernardo Almeida, Vladimir Ya. Shur, and Andrei L. Kholkin

Citation: Appl. Phys. Lett. 109, 162903 (2016); doi: 10.1063/1.4965710

View online: http://dx.doi.org/10.1063/1.4965710

View Table of Contents: http://aip.scitation.org/toc/apl/109/16

Published by the American Institute of Physics

\section{Articles you may be interested in}

Ferroelectric, pyroelectric, and piezoelectric properties of a photovoltaic perovskite oxide Appl. Phys. Lett. 110, 063903063903 (2017); 10.1063/1.4974735

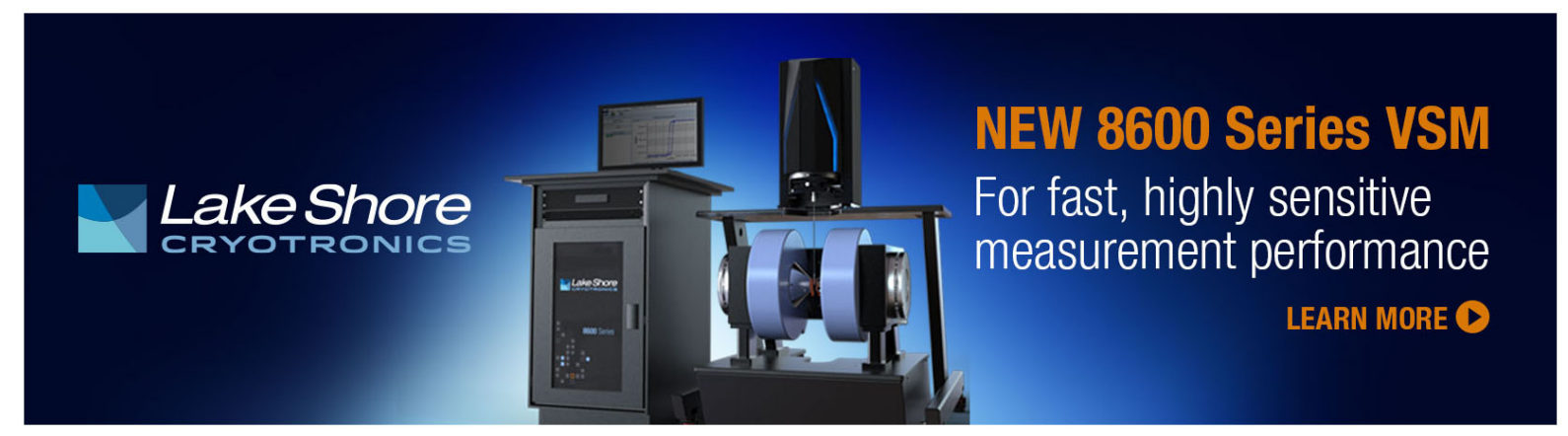




\title{
Probing ferroelectric behaviour in charge-transfer organic meta-nitroaniline
}

\author{
Dmitry Isakov, ${ }^{1, a)}$ Semen Vasilev, ${ }^{2}$ Etelvina de Matos Gomes, ${ }^{1}$ Bernardo Almeida, ${ }^{1}$ \\ Vladimir Ya. Shur, ${ }^{2}$ and Andrei L. Kholkin ${ }^{2,3}$ \\ ${ }^{1}$ University of Minho, Centre of Physics, Campus de Gualtar, 4710057 Braga, Portugal \\ ${ }^{2}$ Institute of Natural Sciences and Mathematics, Ural Federal University, Lenin Ave. 51, Ekaterinburg 620000, \\ Russia \\ ${ }^{3}$ Department of Physics and CICECO Materials Institute of Aveiro, University of Aveiro, \\ 3810193 Aveiro, Portugal
}

(Received 8 May 2016; accepted 7 October 2016; published online 18 October 2016)

\begin{abstract}
Potential ferroelectricity in charge-transfer organic materials is often masked by the intrinsic conductivity. Here, we report the compelling evidence of ferroelectricity in organic $\pi$-conjugated meta-nitroaniline ( $m$-NA) crystals as shown by the local electromechanical measurements using the piezoresponse force microscopy (PFM) technique. $m$-NA is a charge-transfer molecular material with the exceptional optical non-linearity and perceptible conductivity along the crystallographic polar axis. While standard Sawyer-Tower measurements revealed an apparently lossydielectric hysteresis, The PFM switching spectroscopy indicated clear ferroelectric behaviour in this technologically important multifunctional material. Further study of the pyroelectric properties in $m$-NA crystals confirmed their high spontaneous polarization of $18 \mu \mathrm{C} / \mathrm{cm}^{2}$ at room temperature, comparable to the best known organic ferroelectrics. Published by AIP Publishing.

[http://dx.doi.org/10.1063/1.4965710]
\end{abstract}

The recent discovery of room-temperature organic ferroelectric materials ${ }^{1-5}$ and the demonstration of ferroelectricity in bio-organic crystals, for example, amino acids and nucleobases, ${ }^{6,7}$ have stimulated interest in their functional properties related to the presence of spontaneous polarization (piezoelectricity, pyroelectricity, and multiferroic behaviour). The strategy for the crystal engineering of pure organic materials possessing ferroelectricity is based on the careful design of molecular symmetry packing in a polar crystalline structure..$^{8-10}$ However, in many known organic polar materials with pronounced nonlinear-optical, piezoelectric, and pyroelectric properties, the possible ferroelectricity has not been investigated so far due to the lack of suitable methods and masking effect of conductivity.

Meta-nitroaniline $\left(\mathrm{O}_{2} \mathrm{NC}_{6} \mathrm{H}_{4} \mathrm{NH}_{2}, m-\mathrm{NA}\right)$ is a derivative of the push-pull 4-nitroaniline paradigm chromophore with the large molecular first optical hyperpolarizability. In these chromophores, known as donor-acceptor charge transfer compounds, the high hyperpolarizability originates from donoracceptor $\left(\mathrm{NO}_{2} \quad \mathrm{NH}_{2}\right)$ conjugated groups linked through a delocalized $\pi$-electron system along the molecular charge transfer axis. This results in very large electro-optic ${ }^{11,12}$ and nonlinear optical coefficients. ${ }^{13,14}$ The intramolecular charge transfer characteristic of benzene derivative molecules containing electron donor and acceptor substituents is also of interest for photoluminescence applications. ${ }^{15}$ Additionally, $m$-NA crystals possess strong piezoelectricity with a potential to be used in the electromechanical sensors and actuators. Bain et al. reported on large values of piezoelectric coefficients obtained by a direct method: $d_{31}$ and $d_{33}$ of about $31 \mathrm{pm} / \mathrm{V}$ and $7 \mathrm{pm} / \mathrm{V}$, respectively. ${ }^{16}$ Lattice distortions measured by the synchrotron X-ray multiple diffraction technique

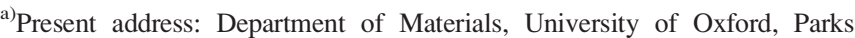
Road, Oxford OX1 3PH, United Kingdom. Electronic mail: dmitry.isakov@ materials.ox.ac.uk.
}

under an applied electric field proved these coefficients to be as high as $165 \mathrm{pm} / \mathrm{V}$ and $103 \mathrm{pm} / \mathrm{V} .^{17}$

The synchrotron X-ray multiple diffraction technique also revealed a distinct signature of ferroelectricity in the $m$-NA crystal $^{18}$ the hysteresis-like behaviour of the X-ray rocking curve peaks in the plane parallel to $b$-axis was caused by the application of an electric field $E$ with a steeply rising strength along the polar $c$-axis. After a certain threshold electric field, the charge transfer over the donor and acceptor groups in $m$-NA becomes nonlinear due to the coupling of two types of geometrical distortions caused by the elongation of the donor-acceptor conjunction group and its torsional change. These cause the hysteresis and memory effect.

Despite a strong interest in room-temperature ferroelectricity in charge transfer complexes, ${ }^{4}$ the ferroelectric properties of $m$-NA have never been studied by conventional techniques, and at this moment, only the report by Avanci et $a l .{ }^{18}$ substantiates this important property in $m$-NA. This is probably due to the difficulties caused by the presence of a notable conductivity and high dielectric losses, which are common for most organic charge-transfer compounds. ${ }^{19,20}$ These are deleterious for the standard Saywer-Tower ferroelectric hysteresis measurement. ${ }^{21}$ Additionally, charge transfer complex materials commonly possess a low breakdown field, causing crystal damage before the switching of polarization occurs.

Alternatively, the polarization state of the material can be probed by the converse piezoelectric effect, on the basis of the piezoresponse force microscopy (PFM) imaging and spectroscopy techniques that allow to detect an alternating displacement of the surface caused by the applied AC electric field with a high spatial resolution (down to $10 \mathrm{~nm}$ ). ${ }^{22}$ Since the amplitude of the piezoresponse is proportional to the local polarization, the method can be used for the evaluation of ferroelectric properties in general. Furthermore, 
recently this technique has been applied for studying the polar properties in soft biological systems..$^{3,7,23-26}$ In this letter, we demonstrate the evidence of a room temperature ferroelectric behaviour in organic charge-transfer $(m-\mathrm{NA})$ crystals, as shown by PFM.

The crystals of $m$-NA were grown by slow evaporation from acetone solution at room temperature in a dust-free chamber. Transparent optical quality, brown-yellow coloured crystals with a needle-like shape up to $10 \mathrm{~mm}$ length and aspect ratio 1:3 5 (as described elsewhere ${ }^{27}$ ) with elongation along [001] were obtained. This habit was used to determine the $c$-axis direction in bulk crystals. ${ }^{27,28}$ In this morphology, the hydrogen-rich faces containing amino groups are exposed on the (001) plane, while oxygen-rich faces containing nitro groups are exposed on the opposite $(00 \overline{1})$ plane, thus forming a net polarization along [001].

$m$-NA crystals are optically biaxial and possess the orthorhombic crystal lattice with space group $\mathrm{Pca} 2_{1}$ (point group $2 \mathrm{~mm}$ ) and lattice parameters $\mathrm{a}=18.873(2) \AA$, $\mathrm{b}=6.5212(9) \AA$, and $\mathrm{c}=4.9980(7) \AA^{29,30}$ The unit cell consists of four donor-acceptor molecules forming long-range polar chains along [011] and [011] , interlinked by $\mathrm{N} \mathrm{H}$ $\mathrm{N}-\mathrm{H}^{\cdots} \mathrm{N} \mathrm{N}$ interactions, where consecutive molecules are parallel to each other. The three-dimensional structure of $m$-NA can be described as an array of all-parallel polar layers perpendicular to [100] and interconnected by weak $\mathrm{C} \mathrm{H}$... $\mathrm{O}$ bonds. The striking structural feature is a polar supermolecule charge-transfer network along $[00 \overline{1}]{ }^{29,31}$

Figure 1 shows the current-voltage characteristics and frequency dependence of impedance measured in the $m$-NA crystal along the polar axis. The current $I$ increases with increasing voltage $V$ in a slightly non-Ohmic fashion (Fig. 1(a)), indicating possible contribution of shallow traps to the electric conductivity in $m$-NA. ${ }^{30}$ The frequency dependence of the impedance (Fig. 1(b)) is attributed to the high contribution of DC conductivity to the impedance. The obtained electrical conductivity $2.5 \times 10^{10} \mathrm{~S} / \mathrm{m}$ is in a good agreement with the previously reported values. ${ }^{32}$ The conductivity mechanism in $m$-NA is well understood ${ }^{32-34}$ and is associated with a "hop and turn" mechanism representing protons hopping and reorientational motions of the benzene ring and both substituents. As revealed in Ref. 32, the $m$-NA crystals are narrow-band gap semiconductors with a sufficiently small bandgap of $1.77 \mathrm{eV}$ resulting from a very small overlap of molecular orbitals. The largest overlap occurs

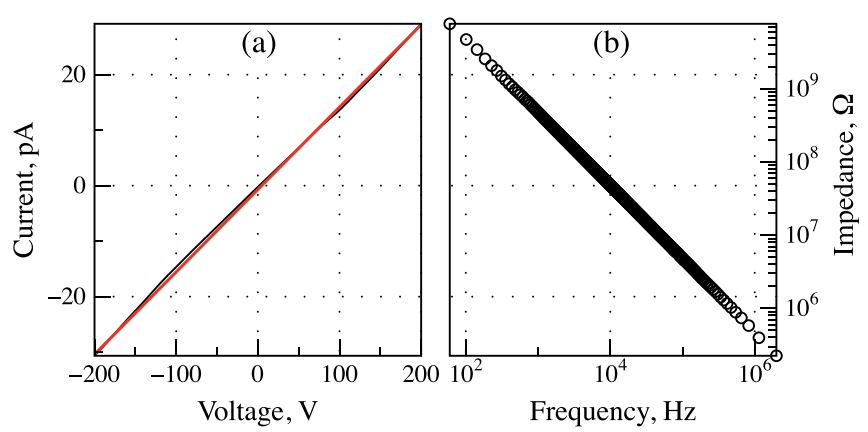

FIG. 1. (a) Current voltage characteristic (red line shows a linear fit of the experimental curve) and (b) frequency dependence of impedance in $m$ NA crystal measured along polar axis. along the polar axis direction that coincides with stacking in $m$-NA crystals. ${ }^{29}$ Thus, one can conclude that the contribution of the conductivity to the dielectric properties of $m$-NA is essential.

Attempts to study the ferroelectric behaviour by the traditional macroscopic Sawyer-Tower technique (with compensation circuit for conductive losses) did not provide a meaningful hysteresis for applied electric fields up to the breakdown field value. Instead, spurious effects due to leakage currents were observed. While the Sawyer-Tower method is based on the detection of accumulated charge resulting from the electrical field induced polarization reversal and thus is prone to conductivity artefacts, the PFM technique is not sensitive to leakage current contribution because the electric field-induced displacement measurements are carried out that are not dependent on the conductivity.

For the study by the PFM technique, the needle-shaped $m$-NA crystals were placed in epoxy resin and then cut perpendicular to the polar $c$-axis. The axis of the scanning cantilever was oriented parallel to the (001) plane. Thus, all studies were conducted on the polar cut of the crystal. Figures 2(a) and 2(b) show the AFM topography of the $2 \times 2 \mu \mathrm{m}^{2}$ area of the $m$-NA crystal surface corresponding to vertical (out-of-plane) piezoelectric response signal. The contrast of the scanned PFM image in Fig. 2(b) represents the structure of domains having opposite directions of the polarization, as proven by the PFM signal phase (the phase difference between bright and dark areas is $180^{\circ}$ ). It should be noted that special precautions were taken to minimize the possible electrostatic effects and the instrumental cross-talk between driving signal and measured response. This has been done by using a cantilever with high stiffness constant and low scan speed operating at frequency $17 \mathrm{kHz}$ that is far below from the resonance of the cantilever. As seen from Figs. 2(a) and 2(b), the obtained features are not related to
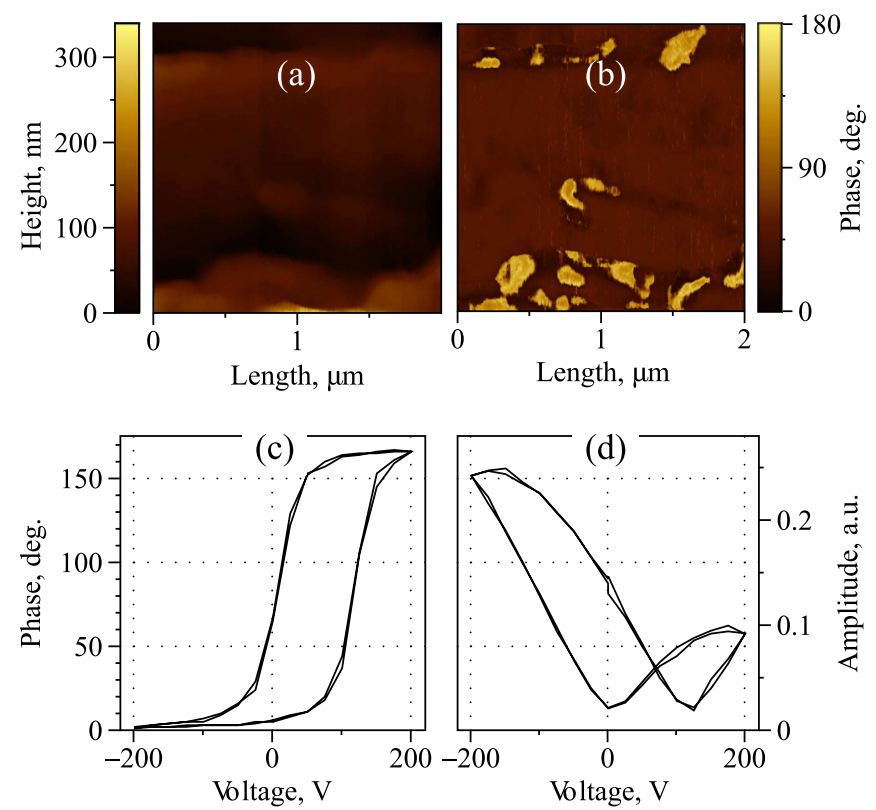

FIG. 2. (a) Topography and (b) domain structure at the surface parallel to the (001) plane, perpendicular to the polar axis in $m$ NA crystal. (c) Phase voltage hysteresis loop and (d) amplitude voltage butterfly loop measured at different points in $m$ NA crystal. 
topographic details and, therefore, can be considered as real ferroelectric domains. The randomness of the domain structure can be attributed to the intrinsic disorder in $m$-NA. ${ }^{35}$

Figures 2(c) and 2(d) show the phase and amplitude hysteresis loops that are representative of the $d_{33}$ piezoresponse $(\propto A \cdot \cos \phi$, where $A$ is the piezoresponse amplitude and $\phi$ is the phase angle) acquired with out-of-plane (longitudinal) PFM. The application of consecutive voltage pulses causes polarization reversal and corresponding variations in the amplitude and sign of the piezoresponse signal. Square phase hysteresis and "butterfly" shape of the amplitude loop (Figs. 2(c) and 2(d), respectively) indicate a switchable out-of-plane polarization component consistent with the unidirectional character of polarization in $m$-NA crystal. The average coercive voltage defined from the local hysteresis (different from the macroscopic coercive one ${ }^{36}$ ) was about $60 \mathrm{~V}$. Such a high voltage could be due to the difficult nucleation of inverse domains or to their strong instability as a result of backswitching. A shift in both phase and amplitude hysteresis loops toward the positive bias voltage could be possibly attributed to the strong charge trapping ${ }^{39,40}$ or bulk screening of depolarization field. It should be noted that the electrostatic contribution due to charge injection (see, for example, Ref. 37) can be excluded because sufficiently stiff cantilevers were used that ensured strong indentation regime of the PFM measurements. ${ }^{38}$ The obtained values for transverse $d_{31}$ (obtained from lateral piezoresponse) and longitudinal $d_{33}$ (derived from vertical displacements) piezoelectric coefficients were found to be $20 \pm 1 \mathrm{pm} / \mathrm{V}$ and $4.0 \pm 0.5 \mathrm{pm} / \mathrm{V}$, respectively. Taken into account that PFM method measures only switchable polarization under local PFM conditions, these values match well with those obtained by a direct method in a bulk crystal. ${ }^{16}$

In order to further investigate ferroelectric properties in $m$-NA crystal, the pyroelectric technique for the measurement of the electric charge induced by the temperature change was used. Figure 3 represents the variation of the pyroelectric current with a change of the ramp temperature obtained by the dynamic pyroelectric measurement. Current measurements were carried out by using an ultra-high input impedance electrometer (Keithley 617) across a shunt

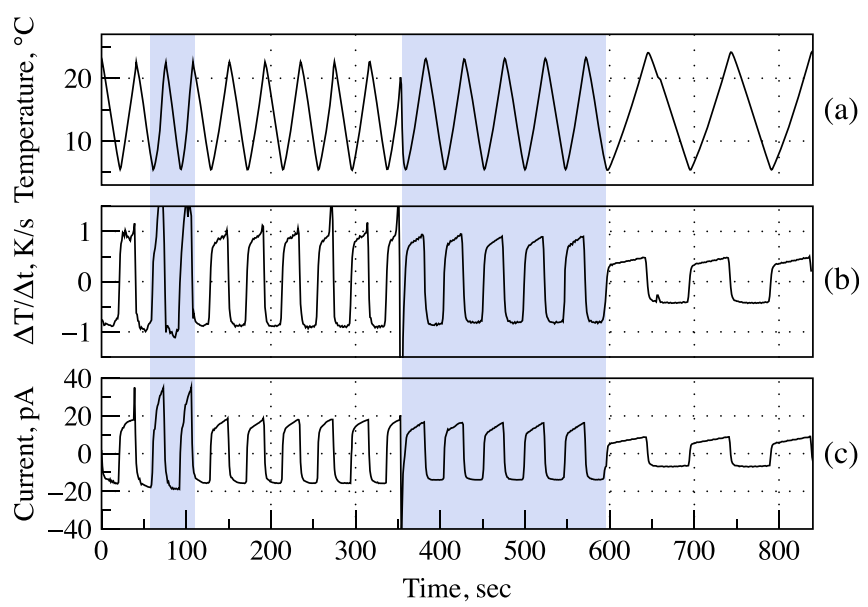

FIG. 3. (a) The temperature cyclic change, (b) derivative, and (c) output pyroelectric current obtained in $m$ NA crystal. Highlighted areas show zones with different temperature derivatives. resistance with the crystal hold under a constant stress to avoid the piezoelectric contribution. The temperature ramp oscillation (Fig. 3(a)) was generated by a Peltier element and was driven with a frequency of about $0.010 .03 \mathrm{~Hz}$ and amplitude of about $17 \mathrm{~K}$ at about $288 \mathrm{~K}$. The pyroelectric response (Fig. 3(c)) is nearly symmetrical with respect to heating/cooling stages and $I(t)$ strictly follows the shape of the temperature derivative $d T / d t$ (Fig. 3(b)). The obtained value of effective pyroelectric coefficient $\rho=4.44 \pm 0.21$ $\times 10^{6} \mathrm{~cm}^{2} \mathrm{~K}^{1}$ is lower than those reported by Asaji and Weiss ${ }^{35}$ acquired by the same method. This deviation may be associated with different measurement conditions and temperatures, and the electrical history of the crystal. The pyroelectric figure of merit $M=\rho / \sqrt{ } \epsilon=2.23$ $\times 10^{8} \mathrm{~cm}^{2} \mathrm{~K}{ }^{1}$ was one order of magnitude higher than that of semiorganic triglycine sulphate crystal. ${ }^{41}$

Figure 4 presents the temperature dependence of spontaneous polarization $P_{s}$ along the polar axis in $m$-NA crystal obtained by the integration of the pyroelectric current during a continuous temperature change with $d T / d t=1 \mathrm{~K} / \mathrm{min}$. It is seen that spontaneous polarization in $m$-NA abruptly disappears above $370 \mathrm{~K}$. This is in a good agreement with the previously reported temperature dependence of dielectric permittivity in $m$-NA crystals, showing a peculiar peak on the dielectric permittivity $\epsilon^{\prime}$ and pronounced drop of $\tan \delta$ at about $373 \mathrm{~K} .{ }^{32,33}$ Additionally, differential scanning calorimetry (DSC) also showed sharp anomalies near $373 \mathrm{~K}$ during cooling. Such anomalies together with the reported large temperature hysteresis in DSC curves are commonly associated with a first-order ferroelectric to paraelectric phase transition. $^{32}$ The disappearance of polarization at $T>370 \mathrm{~K}$ allowed estimating the spontaneous polarization of $m$-NA. The polarization value of $18 \mu \mathrm{C} / \mathrm{cm}^{2}$ obtained in the $m$-NA crystal is among the highest values measured in singlecomponent organic H-bonded crystalline compounds. The highest value of spontaneous polarization of $23 \mu \mathrm{C} / \mathrm{cm}^{2}$ and $21 \mu \mathrm{C} / \mathrm{cm}^{2}$ was reported in a diisopropylammonium bromide (DIPAB) and croconic acid crystals, respectively. ${ }^{5,42}$ Table I summarizes the properties of the $m$-NA crystal in comparison with the data for some other room-temperature organic ferroelectrics.

The polarization of $\mathrm{H}$-bonded ferroelectrics originates from the rearrangement of the $\pi$-conjugated electron system (tautomerism) associated with a reversible and collective proton transfer. As previously proposed in Ref. 34, the mechanism of polarization reversal under applied field in $m$-NA can occur

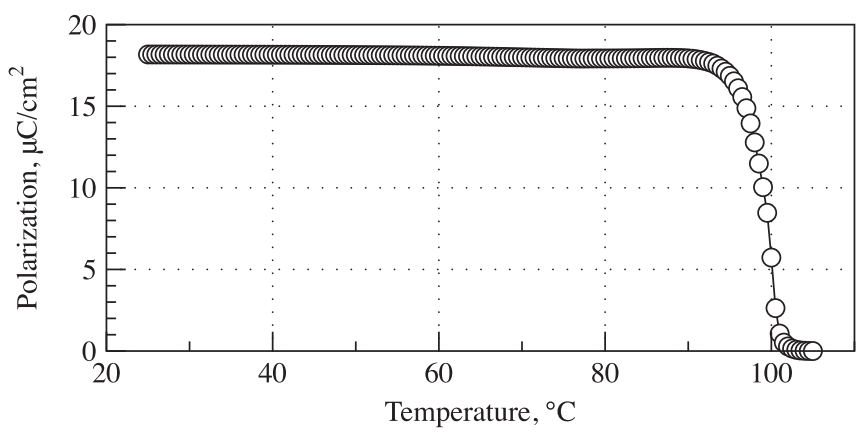

FIG. 4. The temperature dependence of the polarization in $m$ NA crystal. 
TABLE I. Properties of $m$ NA crystals (at room temperature) in comparison with selected high temperature organic ferroelectrics. $P_{s}$ is spontaneous polarization, $T_{c}$ is Curie temperature, $\epsilon_{r}$ is relative dielectric permittivity, $\rho$ is pyroelectric coefficient, and $d_{i j}$ is a component of piezoelectric tensor.

\begin{tabular}{lccccc}
\hline \hline Material & $P_{s}, \mu \mathrm{C} / \mathrm{cm}^{2}$ & $\epsilon_{r}^{\prime}$ & $\rho, \mu \mathrm{C} / \mathrm{m}^{2} \mathrm{~K}$ & $d_{i j}, \mathrm{pV} / \mathrm{m}$ & Ref. \\
\hline$m$ NA & 18.0 & 3.8 & 4.44 & $20\left(d_{31}\right)$ & \\
& & & 7.5 & $31\left(d_{31}\right)$ & 16,35 \\
Croconic acid & 21 & 11 & $55 \pm 20$ & & 5 \\
MBI & 10 & & & & 2 \\
DIPAB & 23 & $\sim 70$ & & $360\left(d_{22}\right)$ & 42 \\
PVDF TrFE & 13 & 7 & 10 & $55\left(d_{33}\right)$ & 43 \\
\hline \hline
\end{tabular}

in a similar way to that described for organic-inorganic hybrid diazabicyclo[2.2.2]octane $\left([\mathrm{dabcoH}]^{+}\left[\mathrm{ReO}_{4}\right]\right.$ ). ${ }^{44}$ Proton hopping connected with a spontaneous polarization along $\mathrm{N} \mathrm{H} \cdots$ $\mathrm{N}$ hydrogen bonding is caused by the rotation and hopping of the anion group. The same switching mechanism was suggested for a single component organic ferroelectric croconic acid. $^{1,4,45}$ It was found that large polarization originates from the charge transfer due to the strong "push-pull" effect of electron-releasing and withdrawing groups along the hydrogen bonds. $^{45}$

This work was supported by the European Regional Development Fund (ERDF) through Programa Operacional Factores de Competitividade (COMPETE: FCOMP-01-0124FEDER-014628, FCOMP-01-0124-FEDER-009457)and FCT Grant No. PTDC/CTMNAN/114269/2009), Pest-C/CTM/ LA0011/013, and by RFBR Grant No. 13-02-90925. This work was developed within the scope of the project CICECO-Aveiro Institute of Materials (POCI-01-0145FEDER-007679, FCT Ref. UID/CTM/50011/2013), financed by national funds through the FCT/MEC and when appropriate co-financed by FEDER under the PT2020 Partnership Agreement, and by Government of the RF (Act 211, Agreement 02.A03.21.0006).

${ }^{1}$ A. S. Tayi, A. K. Shveyd, A. C. H. Sue, J. M. Szarko, B. S. Rolczynski, D. Cao, T. J. Kennedy, A. A. Sarjeant, C. L. Stern, W. F. Paxton, W. W. Wu, S. K. Dey, A. C. Fahrenbach, J. R. Guest, H. Mohseni, L. X. Chen, K. L. Wang, J. F. Stoddart, and S. I. Stupp, Nature 488, 485 (2013).

${ }^{2}$ F. Kagawa, K. Hatahara, K. Kobayashi, R. Kumai, Y. Murakami, Y. Tokura, and S. Horiuchi, Nat. Commun. 3, 1308 (2012).

${ }^{3}$ Y. Liu, Y. Zhang, M. J. Chow, Q. N. Chen, and J. Li, Phys. Rev. Lett. 108, 078103 (2012).

${ }^{4}$ T. A. Tayi, A. Kaeser, M. Matsumoto, T. Aida, and S. I. Stupp, Nat. Chem. 7, 281 (2015).

${ }^{5}$ S. Horiuchi, Y. Tokunaga, G. Giovannetti, S. Picozzi, H. Itoh, R. Shimano, R. Kumai, and Y. Tokura, Nature 463, 789 (2010).

${ }^{6}$ A. Heredia, V. Meunier, I. K. Bdikin, J. Gracio, N. Balke, S. Jesse, A. Tselev, P. K. Agarwal, B. G. Sumpter, S. V. Kalinin, and A. L. Kholkin, Adv. Funct. Mater. 22, 2996 (2012).

${ }^{7}$ I. Bdikin, A. Heredia, S. M. Neumayer, V. S. Bystrov, J. Gracio, B. J. Rodriguez, and A. L. Kholkin, J. Appl. Phys. 118, 072007 (2015).

${ }^{8}$ R. R. Choudhury and R. Chitra, Cryst. Res. Technol. 41, 1045 (2006).
${ }^{9}$ Z. Zikmund, P. Vanek, M. Havrankova, B. Brezina, M. Cermak, and M. Vasa, Ferroelectrics 158, 223 (1994).

${ }^{10}$ G. D'Avino and M. J. Verstraete, Phys. Rev. Lett. 113, 237602 (2014).

${ }^{11}$ J. L. Stevenson and A. C. Skapski, J. Phys. C: Solid State Phys. 5, L233 (1972).

${ }^{12}$ A. Leyderman and Y. Cui, SPIE Proc. 3474, 84 (1998).

${ }^{13}$ G. F. Huang, J. T. Lin, G. Su, R. Jiang, and S. Xie, Opt. Commun. 89, 205 (1992).

${ }^{14}$ V. Krishnakumar and R. Nagalakshmi, Cryst. Growth Des. 8, 3882 (2008).

${ }^{15}$ M. Szostak, B. Kozankiewicz, G. Wójcik, and J. Lipiński, Faraday Trans. 94, 3241 (1998).

${ }^{16}$ M. Bain, N. El Korashy, S. Gilmour, R. A. Pethrick, and J. N. Sherwood, Philos. Mag. Part B 66, 293 (1992).

${ }^{17}$ L. H. Avanci, L. P. Cardoso, S. E. Girdwood, D. Pugh, J. N. Sherwood, and K. J. Roberts, Phys. Rev. Lett. 81, 5426 (1998).

${ }^{18}$ L. H. Avanci, R. S. Braga, L. P. Cardoso, D. S. Galvao, and J. N. Sherwood, Phys. Rev. Lett. 83, 5146 (1999).

${ }^{19}$ D. R. Rosseinsky, J. A. Stephan, and J. S. Tonge, J. Chem. Soc. Faraday Trans. 77, 1719 (1981).

${ }^{20}$ V. R. Nikitenko, A. R. Tameev, and A. V. Vannikov, Org. Electron. 12, 589 (2011).

${ }^{21}$ J. F. Scott, J. Phys.: Condens. Matter. 20, 021001 (2008).

${ }^{22}$ S. V. Kalinin, B. J. Rodriguez, S. Jesse, J. Shin, A. P. Baddorf, P. Gupta, H. Jain, D. B. Williams, and A. Gruverman, Microsc. Microanal. 12, 206 (2006).

${ }^{23}$ F. Kagawa, S. Horiuchi, N. Minami, S. Ishibashi, K. Kobayashi, R. Kumai, Y. Murakami, and Y. Tokura, Nano Lett. 14, 239 (2014).

${ }^{24}$ D. Isakov, D. Petukhova, S. Vasilev, A. Nuraeva, T. Khazamov, E. Seyedhosseini, P. Zelenovskiy, V. Ya. Shur, and A. L. Kholkin, Cryst. Growth Des. 14, 4138 (2014).

${ }^{25}$ D. Denning, S. Alilat, S. Habelitz, A. Fertala, and B. Rodriguez, J. Struct. Biol. 180, 409 (2012).

${ }^{26}$ D. Isakov, E. de, M. Gomes, I. Bdikin, B. Almeida, M. Belsley, M. Costa, V. Rodrigues, and A. Heredia, Cryst. Growth Des. 11, 4288 (2011).

${ }^{27}$ H. X. Cang, W. D. Huang, and Y. H. Zhou, J. Cryst. Growth 192, 236 (1998).

${ }^{28}$ G. Ryu and C. S. Yoon, J. Cryst. Growth 191, 190 (1998).

${ }^{29}$ G. Wójcik and J. Holband, Acta Crystallogr., Sect. A Found. Cryst. 57, 346 (2001).

${ }^{30}$ A. C. Skapski and J. L. Stevenson, J. Chem. Soc., Perkin Trans. 2, 1197 (1973).

${ }^{31}$ W A. E. Goeta, C. C. Wilson, J. A. Autino, J. Ellena, and G. Punte, Chem. Mater. 12, 3342 (2000).

${ }^{32}$ M. M. Szostak, H. Chojnacki, E. Staryga, M. Dluzniewski, and G. W. Bak, Chem. Phys. 365, 44 (2009).

${ }^{33}$ M. M. Szostak, G. Wojcik, J. Gallier, and M. Bertault, Chem. Phys. 229, 275 (1998).

${ }^{34}$ M. M. Szostak and H. Chojnacki, Opt. Mater. 33, 1395 (2011).

${ }^{35}$ T. Asaji and A. Weiss, Zeitschrift fr Naturforschung A 40, 567 (1985), see http://adsabs.harvard.edu/abs/1985ZNatA..40..567A.

${ }^{36}$ A. Wu, P. M. Vilarinho, V. V. Shvartsman, G. Suchaneck, and A. L. Kholkin, Nanotechnology 16, 2587 (2005).

${ }^{37}$ N. Balke, P. Maksymovych, S. Jesse, A. Herklotz, A. Tselev, C. B. Eom, I. I. Kravchenko, P. Yu, and S. V. Kalinin, ACS Nano 9, 6484 (2015).

${ }^{38}$ S. V. Kalinin and D. A. Bonnell, Phys. Rev. B 65, 125408 (2002).

${ }^{39}$ C. K. Wong and F. G. Shin, J. Appl. Phys. 96, 6648 (2004).

${ }^{40}$ X. L. Wang, B. Li, X. L. Zhong, Y. Zhang, J. B. Wang, and Y. C. Zhou, J. Appl. Phys. 112, 114103 (2012).

${ }^{41}$ H. M. Choe, J. H. Judy, and A. van der Ziel, Ferroelectrics 15, 181 (1977).

${ }^{42}$ D. W. Fu, H. L. Cai, Y. Liu, Q. Ye, W. Zhang, Y. Zhang, X. Y. Chen, G. Giovannetti, M. Capone, J. Li, and R. G. Xiong, Science 339, 425 (2013).

${ }^{43}$ G. Bai, R. Li, Z. G. Liu, Y. D. Xia, and J. Yin, J. Appl. Phys. 111, 044102 (2012).

${ }^{44}$ M. Szafrański, A. Katrusiak, and G. McIntyre, Phys. Rev. Lett. 89, 215507 (2002).

${ }^{45}$ Y. Cai, S. Luo, Z. Zhu, and H. Gu, J. Chem. Phys. 139, 044702 (2013). 